\title{
Transient stability analysis of electric energy systems via a fuzzy ART-ARTMAP neural network
}

\author{
Wagner Peron Ferreira*, Maria do Carmo G. Silveira, AnnaDiva P. Lotufo, Carlos. R. Minussi \\ Department of Electrical Engineering, São Paulo State University (UNESP), P.O. Box 31, 15385-000, Ilha Solteira, SP, Brazil
}

Received 12 September 2005; accepted 12 September 2005

Available online 6 January 2006

\begin{abstract}
This work presents a methodology to analyze transient stability (first oscillation) of electric energy systems, using a neural network based on ART architecture (adaptive resonance theory), named fuzzy ART-ARTMAP neural network for real time applications. The security margin is used as a stability analysis criterion, considering three-phase short circuit faults with a transmission line outage. The neural network operation consists of two fundamental phases: the training and the analysis. The training phase needs a great quantity of processing for the realization, while the analysis phase is effectuated almost without computation effort. This is, therefore the principal purpose to use neural networks for solving complex problems that need fast solutions, as the applications in real time. The ART neural networks have as primordial characteristics the plasticity and the stability, which are essential qualities to the training execution and to an efficient analysis. The fuzzy ART-ARTMAP neural network is proposed seeking a superior performance, in terms of precision and speed, when compared to conventional ARTMAP, and much more when compared to the neural networks that use the training by backpropagation algorithm, which is a benchmark in neural network area.
\end{abstract}

(c) 2005 Elsevier B.V. All rights reserved.

Keywords: Adaptive resonance theory; ART-ARTMAP

\section{Introduction}

This work aims to present a methodology to transient stability analysis of electric energy system by artificial neural networks. The stability analysis consists of evaluating the effects proceeding of perturbations that cause great and undesirable oscillations on the angles of synchronous machines. This analysis can be realized by solving non-linear differential equations, that describe the movement of the system - synchronous machine oscillation equation - and, after by the analysis of the evolution of the angular position of each synchronous machine during time (simulation) [1]. The techniques used for the simulation are precise and present no restrictions to the type of the model employed. However, it is necessary to effectuate complex calculus that consists of solving a set of algebraic and non-linear differential equations and examining the oscillation curves that are obtained, increasing the time dispensed to conclude the anal-

\footnotetext{
* Corresponding author.

E-mail addresses: wagner@dee.feis.unesp.br (W.P. Ferreira), carmo@feis.unesp.br (M.d.C.G. Silveira), annadiva@dee.feis.unesp.br (A.P. Lotufo), minussi@dee.feis.unesp.br (Carlos.R. Minussi).
}

ysis. An alternative proceeding consists of obtaining the analysis without solving the differential equations. In this way, there is the Lyapunov direct method (LDM) [2], which results are considered satisfactory, principally when it employed the classical method [2-4]. Taking into account the great quantity of the defects to be analyzed, the complexity and the great dimension of the modern electric systems, the simulation, as well as the Lyapunov direct method, are alternatives that, not yet, offer no conditions to applications in real time. New approaches of analysis, based on artificial intelligence, in special the neural networks [5], are presented in the literature aiming to overcome these difficulties, principally concerning to velocity. Therefore, this work investigates the application of artificial neural networks (ANN) on the diagnosis of transient stability analysis of electric energy systems. The transient stability analysis by neural networks, is in general effectuated employing the feedforward networks, with the training based on the backpropagation [6]. This technique is efficient, being considered in the literature, as a benchmark in terms of precision. However, the processing time is relatively high.

This way, in this work it is investigated the use of the fuzzy neural ART-ARTMAP network [7]. The ART-ARTMAP 
network is an architecture based on the supervised training for multi-dimensional mappings (multi-input/multi-output), being composed of three ART modules [8], and an inter-ART module. The ART neural network family has the characteristic of stability (capacity of learning without adjusting the weights) and plasticity (capacity of continue learning with the inclusion of new patterns without loosing the memory in relation to the previous patterns). Considering these characteristics, the expectation is that the network presents a superior performance when compared to the traditional backpropagation [6]. The ARTMAP neural networks, as well as the ART networks, are capable to include innovations that can produce better results [9]. The ART module is used to classify the input vectors (analog data), corresponding to the active and reactive nodal power in different categories and to convert them in binary information $\left\{\boldsymbol{P}^{\text {bin }}, \boldsymbol{Q}^{\text {bin }}\right\}$, by a processing module active code/binary code [7]. The fuzzy ARTMAP input module is constituted by the set $\left\{\boldsymbol{P}^{\text {bin }}, \boldsymbol{Q}^{\text {bin }}\right\}$, added to the binary information referred to fault conditions and the electric network topology. Thus, the neural ARTMAP network receives only binary data that represent a favorable situation for applications in large systems. This represents a reduction on the computational effort necessary to realize the training and an improvement on the quality of the analysis, when compared to other neural networks.

It is emphasized that, in the specialized literature, there are few references that deal with the stability analysis problem using neural networks [10]. In almost of these references, the transient stability analysis problem is formulated aiming the determination of the critical times for short circuit faults. References [10-12] use neural networks with the training by the backpropagation algorithm. In reference [13], it is used the backpropagation algorithm with a fuzzy controller, which adjusts the training rate, to reduce the number of cycles and the execution time in the training phase. There are other references, in special, that deal with problems associated to the electric power systems by neural networks using the ART concept. In references [14-16], the transient stability analysis problem solved by neural networks is proposed using a fuzzy ARTMAP neural network, considering the variation of the load and generation by proportionality, being this the majority of the solutions adopted in the available references. To illustrate the proposed methodology results are presented considering a multi-machine system.

\section{System model}

Considering an electric energy system composed of ns synchronous machines, the dynamic behavior of the $i$-th synchronous machine, related to the center of angles [2] is described by the following differential and non-linear algebraic Eqs. (2)-(4):

$$
\begin{aligned}
& M_{i} \frac{\mathrm{d}^{2} \theta_{i}}{\mathrm{~d} t^{2}}-P_{i}(\boldsymbol{\theta})=0 \\
& P_{i}(\boldsymbol{\theta})=\frac{\operatorname{Pm}_{i}-\mathrm{Pe}_{i}-\left(M_{i} \text { PCA }\right)}{\mathrm{MT}}, \quad i \in N .
\end{aligned}
$$

where $M_{i}=2 H_{i} / \omega_{\mathrm{s}} ; \omega_{\mathrm{s}} \underline{\Delta}$ synchronous velocity $\left(=2 \pi f_{0}\right) ; H_{i}=$ inertial constant $(\mathrm{s}) ; f_{0}$ is the nominal frequency of the system $(\mathrm{Hz}) ; \theta_{i} \Delta$ rotor angle of the $i$-th synchronous machine in relation to the center of angles (electrical radians) $\left(=\delta_{i}-\delta_{0}\right)$; $\delta_{i}$ is the rotor angle of the $i$-th synchronous machine in relation to a machine that rotates in synchronous velocity (electrical radians); $\delta_{0}=\sum_{j \in N} M_{j} \delta_{j} / \mathrm{MT} ; \boldsymbol{\theta}=\left[\theta_{1}, \theta_{2}, \ldots, \theta_{\mathrm{ns}}\right]^{\mathrm{T}} ; \varpi=$ $\left[\varpi_{1}, \varpi_{2}, \ldots, \varpi_{\mathrm{ns}}\right]^{\mathrm{T}} ; \mathrm{Pm}_{i}$ is the input mechanical power $(\mathrm{pu})$; $\mathrm{Pe}_{i}$ is the output mechanical power (pu); PCA $\underline{\Delta}$ accelerating power of the center of the angles $\left(=\sum_{j \in N}\left(\mathrm{Pm}_{j}-\mathrm{Pe}_{j}\right)\right)$; $\mathrm{MT}=\sum_{j \in N} M_{j} ; N \underline{\Delta}$ index set of the synchronous machines $=(\{1,2, \ldots, \mathrm{ns}\}) ; \mathrm{ns}$ is the quantity of synchronous machines.

\section{Transient stability analysis}

The transient stability analysis of electric power systems, considering a contingency of index $r$, can be realized using the security margin criterion [17]:

$\mathcal{M}_{r}=\frac{E_{\mathrm{crit}_{r}}-E_{\mathrm{e}_{r}}}{E_{\mathrm{crit}_{r}}}$

where $E_{\mathrm{crit}_{r}}$ is the total critical energy of the system; $E_{\mathrm{e}_{r}}$ is the total energy of the system referred to the fault elimination time.

The critical energy ( $\left.E_{\text {crit }}\right)$ and the critical time (crit) can be determined using a series of solutions available on the specialized literature, as an example, the method potential energy boundary surface (PEBS) $[18,19]$. It is observed that for the classical method, the PEBS method gives the same results as the simulation [19]. The total energy, referred to the system defined by Eqs. (1) and (2), is given by [2]:

$E(\boldsymbol{\theta}, \boldsymbol{\omega})=E_{\mathrm{c}}(\boldsymbol{\omega})+E_{\mathrm{p}}(\boldsymbol{\theta})$

where

$E_{\mathrm{c}}(\omega)=\frac{1}{2} \sum_{i \in N} M_{i} \omega_{i}^{2}$ (kinetic energy)

$E_{\mathrm{p}}(\theta)=-\sum_{i \in N} \int_{\theta_{i}^{p}}^{\theta_{i}} P_{i}(\theta) \mathrm{d} \theta_{i}$ (potential energy)

In this work, it is used the iterative PEBS method [19]. Nevertheless, the developed methodology (by fuzzy ART-ARTMAP network) is not dependent of the PEBS method, and another method can be used, since some stability index be provided.

Then, the transient stability of the $r$-th contingency can be inferred by the security margin on the following way [17]:

- if $\mathcal{M}_{r} \geq 0$, then, the system is considered stable;

- if $\mathcal{M}_{r} \leq 0$, then, the system is considered unstable.

\section{Transient stability analysis: solution proposed by neural networks}

In this section, it is established the input and the output of the neural network. The information are provided by a computational program of transient stability analysis (Simul) [17] that, from electrical network data, considering a list of contingencies 
(three phase short circuit), come the associated security margin values. The Simul program is a computational implementation based on numeric integration of differential equations (Eq. (1)) and on the PEBS method, microcomputer version.

\subsection{Input stimulus}

The proposed neural structure aims to analyze the electric energy system transient stability, that corresponds to the security margin determination, considering faults from a three phase short circuit with outage of a transmission line. The input pattern vectors of the neural network are defined as [12,20]:

$\boldsymbol{X}_{r}=\left[\boldsymbol{P}^{\mathrm{T}} \boldsymbol{Q}^{\mathrm{T}} \boldsymbol{\Lambda}_{r}^{\mathrm{T}}\right]^{\mathrm{T}}$

where $\boldsymbol{X}_{r}$ is the pattern vector referred to the $r$-th contingency; $\boldsymbol{P}=\left[\begin{array}{llll}P_{1} & P_{2} & \ldots & P_{\rho}\end{array}\right]^{\mathrm{T}} ; \boldsymbol{Q}=\left[\begin{array}{lll}Q_{1} & Q_{2} & \ldots Q_{\rho}\end{array}\right]^{\mathrm{T}} ; P_{i}$ is the active power of the $i$-th bus of the system; $Q_{i}$ is the reactive power of the $i$-th bus of the system; $\boldsymbol{\Lambda}_{v}$ is the vector that contains the information about the $\nu$-th contingency represented in binary code.

In this case, considering the classical model for the first oscillation transient stability analysis [3], it is supposed that only the active and reactive power vectors are necessary. The topology of the network and the other parameters (inertia constant, transient reactance, etc.) are maintained constant, consequently only the causal variables (active and reactive power) and the parameters that express alterations on the network (representation of the contingencies) are used. It is emphasized that the state of the system (angles and velocities) are elements that are consequences of this process, i.e. they are function of the power (active and reactive) and of the type and circumstance of the fault.

\subsection{Output stimulus}

The stimulus to be applied to the ARTb module of the ARTARTMAP neural network are the security margins corresponding to the input pattern vectors (ARTa module) described by Eq. (7). The strategy proposed in this work, is to use intervals of security margin where the contingencies are. This form permits to represent the contingencies by a binary code and reduce the quantity of classes on ARTb module, that is an adequate way of working with ART-ARTMAP network, providing a faster training and the analysis more reliable (there is always a guarantee of obtaining a solution). It used the concept of security margin displacement effort [17] to establish these intervals:

$\vartheta=\left(\frac{1-\mathcal{M}^{\mathrm{A}}}{1-\mathcal{M}^{\mathrm{B}}}\right)-1$

where $\vartheta$ is the security margin displacement effort; $\mathcal{M}^{\mathrm{A}}$ is the value of the initial security margin of the interval; $\mathcal{M}^{\mathrm{B}}$ is the value of the final security margin of the interval.

Thus, from Eq. (8), it can be established the following recurrence equation (difference equation):

$\mathcal{M}(k+1)=g \mathcal{M}(k)+h$

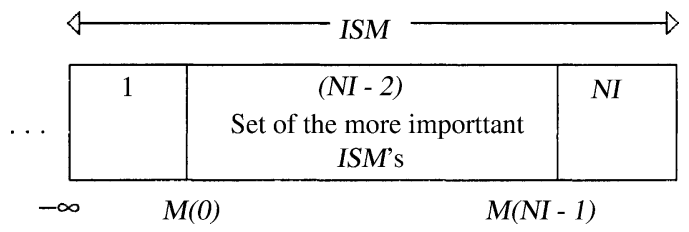

Fig. 1. Representation of the ISMs on the interval $(-\infty, 1]$.

where $g \Delta 1 /\left(1+\vartheta_{\text {esp }}\right) ; h=1-g ; \vartheta_{\text {esp }}$ is the value of $\vartheta$ specified according to the established criterion.

Eq. (9) is obtained from Eq. (8) substituting $\mathcal{M}^{\mathrm{A}}$ and $\mathcal{M}^{\mathrm{B}}$ by $\mathcal{M}(k)$ and $\mathcal{M}(k+1)$, respectively. Effectuating the discrete variation on time $k$, between 0 and $\infty$, it is obtained a discrete curve comprehended between the initial security margin value $\mathcal{M}(0)$ (start value) and the final security margin $\mathcal{M}(\infty)=1$.

Therefore, the interval, in function of the concept of the security margin interval, can be defined as:

$\operatorname{ISM}(k) \underline{\Delta}\left(\mathcal{M}_{\text {inferior }}(k), \mathcal{M}_{\text {superior }}(k)\right), \quad$ for $k=1,2,3, \ldots, \mathrm{NI}$

where $\operatorname{ISM}(k)$ is the $k$-th interval of the security margin and NI is the number of the intervals of the ISMs. [20]:

The functions $\mathcal{M}_{\text {inferior }}(k)$ and $\mathcal{M}_{\text {superior }}(k)$ are defined as

$\mathcal{M}_{\text {superior }}(k)=1-v_{\mathrm{s}} \exp (\phi k)$

$\mathcal{M}_{\text {inferior }}(k)=1-v_{i} \exp (\phi k)$

where $v_{\mathrm{s}}=\{1-\mathcal{M}(0)\} /\left(1+\vartheta_{\text {esp }}\right) ; \phi=-\ln \left(1+\vartheta_{\text {esp }}\right) ; \ln$ is the Neperian logarithm.

The number of interval NI determines the number of bits necessary to the representation of the output values of the neural network. For example, if it is desired NI between 8 and 15, it is necessary 4 bits.

It is assigned $-\infty$ to $\mathcal{M}_{\text {inferior }}(1)$, only to be evident, due to practical situations where the operative conditions with security margin inferior to -10 are rare. Contingencies with security margins inferior to -3 and superior to 0.9 can be considered very unstable and very stable, respectively. In Fig. 1, it is illustrated the distribution of the intervals of the security margin. It is observed that the central set composed by (NI - 2) ISMs corresponds to the more plausible occurrence situation. ISM(1) and ISM(NI) show contingencies very unstable and very stable, respectively.

The relation with the number of the interval $k$ of the security margin ISM and the parameter $\vartheta$ (security margin displacement effort) can be expressed by [19]:

$\vartheta=\{\Gamma(k)\}^{\psi(k)}-1$

where

$\Gamma(k) \underline{\Delta}\left\{\frac{\left(1-\mathcal{M}_{\text {superior }}(k)\right.}{1-\mathcal{M}(0)}\right\}$

$\psi(k) \underline{\Delta} \frac{-1}{k-1}$. 
For determining the parameter $\vartheta$, considering $k$ corresponding to the last but one ISM, i.e. $k=\mathrm{NI}-1$, it is enough to substitute this value on Eqs. (13)-(15), that define the associated parameters:

$\vartheta_{\text {esp }}=\{\Gamma(\mathrm{NI}-1)\}^{\psi(\mathrm{NI}-1)}-1$

where

$\Gamma(\mathrm{NI}-1) \underline{\Delta}\left\{\frac{\left(1-\mathcal{M}_{\text {superior }}(\mathrm{NI}-1)\right.}{1-\mathcal{M}(0)}\right\}$

$\psi(\mathrm{NI}-1) \underline{\Delta} \frac{-1}{\mathrm{NI}-2}, \quad$ for $k=1,2,3, \ldots, \mathrm{NI}$.

It is observed that the relation with the number of intervals $\mathrm{NI}$ and the number of bits can be expressed by:

$\mathrm{NI}=2^{(n \text { bits })}-1$

where $n$ bits is the number of bits used to the representation of the output variable (ISM).

The intervals ISMs keep great correlation with the vigilance parameter $(\rho)$ of the neural network ART architecture. This propriety, therefore, is characterized as a very important result in treating ARTMAP neural networks, principally if it is adopted the binary representation on the output as a way of representing an analog quantity. The solution quality control is established by the parameter $\vartheta_{\text {esp }}$, and it is also implicit the control of the vigilance parameter $\rho$, i.e. the classes will be formed with more quality. It is observed that a representation with 6 bits (until 63 intervals), taking the first interval defined as $(-\infty,-10]$, the curve ISM between -10 and 1 (or close to 1) presents a behavior that approaches an analog curve:

$\operatorname{ISM}(k) \cong \mathcal{M}_{\text {superior }}(k) \cong \mathcal{M}_{\text {inferior }}(k)$

Considering the results that were explained, it must be given priority to the values of $\mathcal{M}$ comprehended between -3 and 0.9 . A good practical alternative is to adopt 15 intervals $(\mathrm{NI}=15)$, i.e. the output is represented by 4 bits. Considering $\mathcal{M}(0)=-3$, $\mathcal{M}_{\text {superior }}(14)=0.9191$ and using Eqs. (16)-(18), it is obtained $\vartheta_{\text {esp }}=0.35$. This representation is illustrated on Fig. 2 .

\section{Neural network proposed}

The ARTMAP neural network is an architecture where the training is realized in a supervised and auto-organized way. The purpose is to approximate non-linear multi-dimensional functions. This schema is adequate to solve a series of complex problems, emphasizing the electric power system transient stability analysis, that is the problem approached in this work. The importance of employing this system is referred to the characteristics of stability and plasticity, besides offering very fast answers (faster training), as already mentioned. However, the neural networks of the ART family present some operational difficulties: great sensibility to the network parameters (vigilance parameter, etc.) and the precision of the analysis. The parameter sensibility is solved, or the effects attenuated, by using a set of training data constituted in a balance way, and by employing new conceptions of training and architecture of ART neural networks

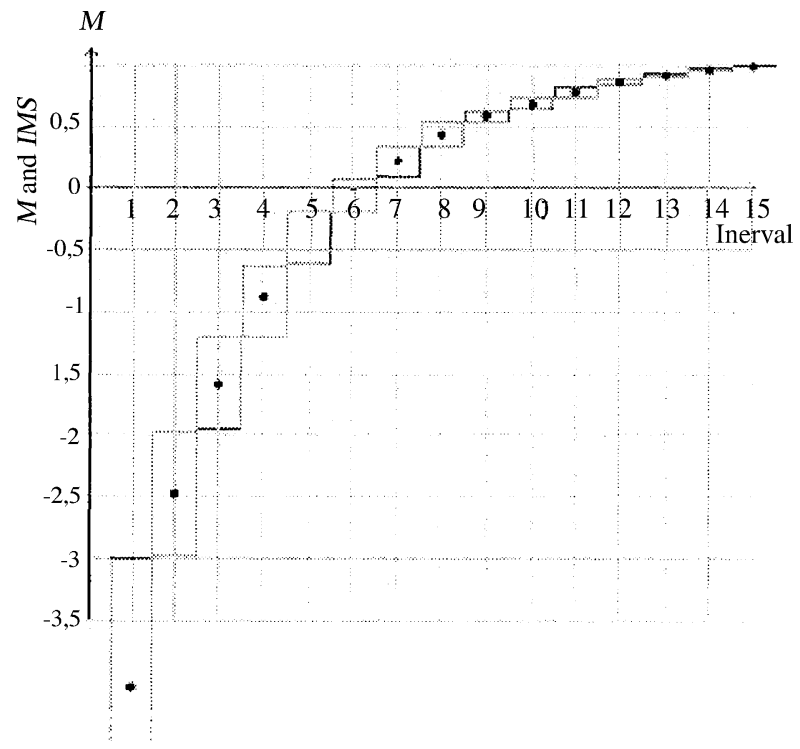

Fig. 2. Graphic of ISM and of $\mathcal{M} \in(-\infty, 1], \vartheta_{\text {esp }}=0.35$ and $\mathcal{M}(0)=-3$.

[9]. In the same way, several proposals are presented on the specialized literature aiming to solve the problem of imprecision. Part of the imprecision is a consequence, principally of the mechanism of the choice of the categories and of the vigilance test.

Therefore, in this work it is proposed a neural system, which objective is to solve, or at least, to reduce the imprecision of the analysis results, by a mechanism that seeks using the analog and the binary data disconnectedly. This used schema is based on a fuzzy ART module, that receives the analog data, producing on the output, the information characteristic of the ART networks, that are the active codes (discrimination of the categories). These categories are processed and transformed in binary information that are added to the other information associated to the problem (binary data), forming the input vector of the ARTMAP module. The ARTMAP neural network output is also binary. The formulation based on binary input and output gives to the ARTMAP neural network more precision, and the use of a modular (ARTARTMAP) architecture [7] provides velocity when compared to the use of a single ARTMAP network (in this case the input data are binary and analog). It is emphasized that this conception preserves the characteristic of plasticity that with no doubt, is one of the principal characteristics of the ART networks.

This allows the implementation of the continuous training, constituting an important resource on the electrical power system operation context. It is also emphasized that these two modules (ART and ARTMAP) correspond to the fuzzy alternative formulation [8].

In Fig. 3, it is shown the fuzzy ART-ARTMAP neural network. It is observed that the input of ART module corresponds to the vector $\boldsymbol{a}^{\text {an }}$ (analog). This is a non-supervised network, i.e. the training is effectuated using only the input data. The output is provided containing the classes (active codes) associated to the input patterns. Through a processing module, these classes are converted in a set of binary data $Z^{\text {bin }}$. The other analog data $\left(\boldsymbol{\Lambda}^{\text {an }}\right)$ are converted in binary data $\left(\boldsymbol{\Lambda}^{\text {bin }}\right)$. This vector is added to the $\boldsymbol{Z}^{\text {bin }}$ vector, composing the input of the ARTMAP module. 


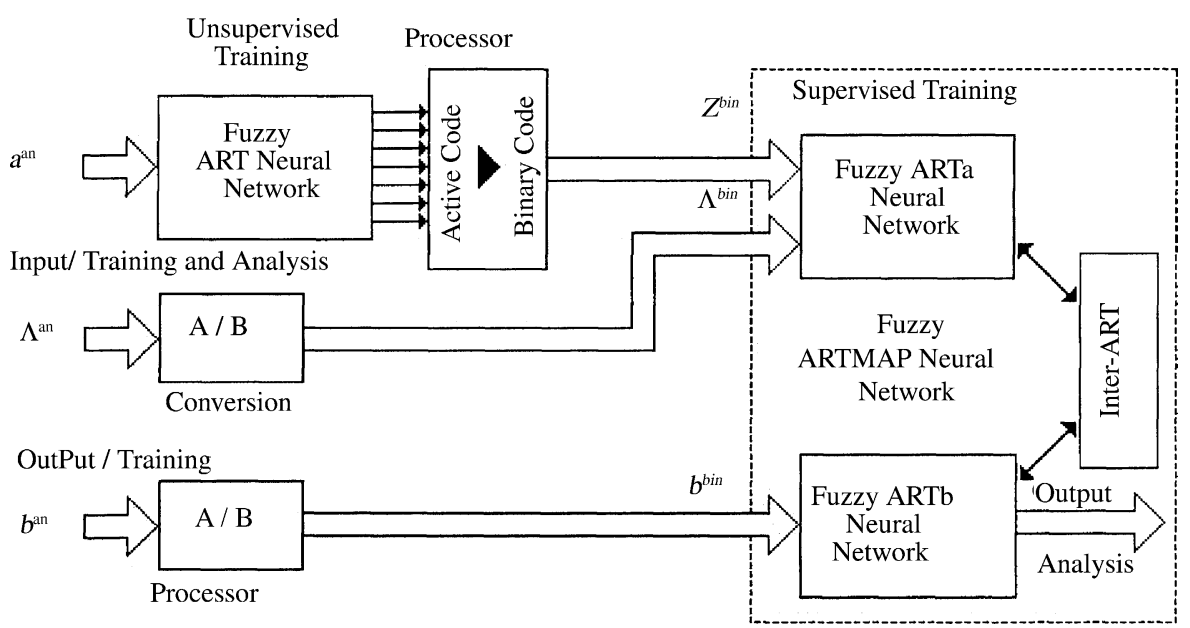

Fig. 3. Fuzzy ART-ARTMAP neural network.

The output, for tests and training, according to Section 4.2, is processed and transformed in binary data.

Following, it is presented the basic formulation of the fuzzy ART neural network and the principal details of the fuzzy ARTMAPN neural network.

\section{Fuzzy algorithm}

The fuzzy ART network algorithm consists, basically of on the following steps $[8,14]$ :

Step 1. Initialization of the weights and choice of the parameters

Initially, the weights and the other parameters of the fuzzy ART neural network are arbitrated on the following way:

$w_{i j}=1, \quad \forall i, j$

$\rho \in[0,1], \alpha>0$ and $\beta \in[0,1]$;

where $\rho$ is the vigilance parameter; $\alpha$ is the choice parameter; $\beta$ is the training parameter.

Step 2. Normalization process

The proliferation category is avoided on the fuzzy ART neural network if the input are normalized:

$Z=\frac{(a)}{|a|}$

where $\boldsymbol{a}$ is the input vector; $\boldsymbol{Z}=\left[\begin{array}{llll}Z_{1} & Z_{2} & \ldots & Z_{m}\end{array}\right]$ (normalized); $|\cdot|=$ norma function.

Step 3. Complementar codification

A new pattern $\boldsymbol{Z}$, where each component $Z_{i}$ is a positive real number pertaining to the interval $[0,1]$, has a complementary codification. This provides an input vector $I$ with MA elements, on the following way:

$\boldsymbol{I} \underline{\Delta}\left[\begin{array}{ll}\boldsymbol{Z} & \boldsymbol{Z}^{\mathrm{c}}\end{array}\right]=\left[\begin{array}{lll}Z_{1} Z_{2} \ldots Z_{m} & Z_{1}^{\mathrm{c}} Z_{2}^{\mathrm{c}} \ldots Z_{m}^{\mathrm{c}}\end{array}\right]$

where $Z_{i}^{\mathrm{c}}=1-Z_{i}$; MA $\underline{\Delta}$ quantity of neurons in $\mathrm{F}_{1}(=2 \mathrm{~m})$.
This complementary codification is a normalization rule that preserves the amplitude of the information [8]:

$|\boldsymbol{I}|=\sum_{i=1}^{m} Z_{i}+\left(m-\sum_{i=1}^{m} Z_{i}\right)=m$.

Step 4. Choice of the categories

The $T_{j}$ function is calculated for each neuron $j$ using the choice function (on activity $\mathrm{F}_{2}$ ):

$T_{j}=\frac{\left|\boldsymbol{I} \wedge \boldsymbol{w}_{j}\right|}{\alpha+\left|\boldsymbol{w}_{j}\right|}$,

where $\wedge$ is the fuzzy operator AND defined by [5]: $(\boldsymbol{p} \wedge \boldsymbol{q})_{i} \Delta \min$ $\left(p_{i}, q_{i}\right)$, with $\boldsymbol{p}$ and $\boldsymbol{q} \in \mathfrak{R}^{L}$,

$|\boldsymbol{p}| \underline{\Delta} \sum_{i=1}^{L}\left|p_{i}\right|$

The choice of the category is considered when, at least one neuron in $\mathrm{F}_{2}$ become active. The chosen category is, then, indexed by the index $J$ on the following way:

$T_{J}=\max \left\{T_{j}, j=1, \ldots, \mathrm{NA}\right\}$

Step 5. Vigilance test

The resonance occurs when the function:

$\left|\frac{\boldsymbol{I} \wedge \boldsymbol{w}_{J}}{\boldsymbol{I}}\right|$

referred to the chosen category satisfies the vigilance criterion:

$\left|\boldsymbol{I} \wedge \boldsymbol{w}_{J}\right| \geq \rho|\boldsymbol{I}|$

Then, the training is realized according to the instructions shown in Step 6. Otherwise, it is said that the vigilance criterion failed to the chosen category. In this case, the value of the chosen function $T_{J}$ is set equal to zero. This represents a total exclusion of the neuron $J$ on the competition process for the current pattern vector (that one where is being processed the 
associated training). Thus, a new index $J$ is chosen using Eq. (24). The seeking process continues until the index $J$ satisfies Eq. (26).

Step 6. Adaptation of the weights

Finalizing the seeking process, the weight vector $\boldsymbol{w}_{J}$ is adapted according to the following equation [8]:

$\boldsymbol{w}_{J}^{\text {new }}=\beta\left(\boldsymbol{I} \wedge \boldsymbol{w}_{J}^{\text {old }}\right)+(1-\beta) \boldsymbol{w}_{J}^{\text {old }}$

It is observed that the fast training corresponds to the adoption of $\beta=1$. With $\beta<1$ the training is slow. The pattern vectors must be presented to the ART neural network in a random way [8].

In relation to the ARTMAP network, the modules ARTa and ARTb have the same structure of the ART neural network described previously, except when a basic vigilance is used to control the system. The weight matrices associated to the modules ARTa $\left(\boldsymbol{w}^{a}\right)$ and $\operatorname{ARTb}\left(\boldsymbol{w}^{b}\right)$, as well as in relation to the Inter-ART ( $\boldsymbol{w}^{a b}$ ) are initiated with values equal to 1 , i.e. every activity is inactive. These activities are activated while occurs the resonance with the input and output patterns. Every time that the input pairs $(\boldsymbol{a}, \boldsymbol{b})$, associated to the modules ARTa and $\mathrm{ART} b$, are confirmed (the inputs $\boldsymbol{a}$ and $\boldsymbol{b}$ referred to the active categories $J$ and $K$, respectively), the weights $\boldsymbol{w}^{a}, \boldsymbol{w}^{b}$ and $\boldsymbol{w}^{a b}$ must be adapted using Eq. (27) and:

$\boldsymbol{w}^{b} K^{\text {new }}=\beta\left(\boldsymbol{I} \wedge \boldsymbol{w}^{b} K^{\text {old }}\right)+(1-\beta) \boldsymbol{w}^{b} K^{\text {old }}$

$\boldsymbol{w}^{a b} J K^{\text {new }}=1$

\section{Application}

In this section, it is presented the results obtained by the proposed method (fuzzy ART-ARTMAP neural network), considering a system composed of 10 synchronous machines, 45 busses and 72 transmission lines, as shown in Fig. 2. This system corresponds to a possible configuration of south Brazil system. Then, the results are presented, comparing the results obtained by neural networks and those produced by simulation (hybrid methodology: PEBS iterative method [19], that is a reference in precision, considering the classical model).

In these studies, the faults are considered three phase faults with elimination time $(t c h)$ equal to nine cycles $(0.15 \mathrm{~s})$ with outage of transmission lines. The network training is realized using a set of generation and load profiles and respective security margins associated to a contingency set. Each profile corresponds to a generation dispatch, in relation to the base case, effectuated in a random way to attend the demand, also established in a random way in each bus. The universe of the generation and the load variation is comprehended between 80 and $130 \%$ in relation to the base case of the system. Therefore, each profile is generated considering a percentile variation around the nominal state (base case) and a determined seed for the generation of the random sequence. Thus, for a same percentile, different seeds generate different generation dispatches and different load profiles. This proceeding generates an adequate set of patterns for the training phase. The comparative analysis with the obtained results by neural networks and by hybrid simulation [19] is satisfactory.

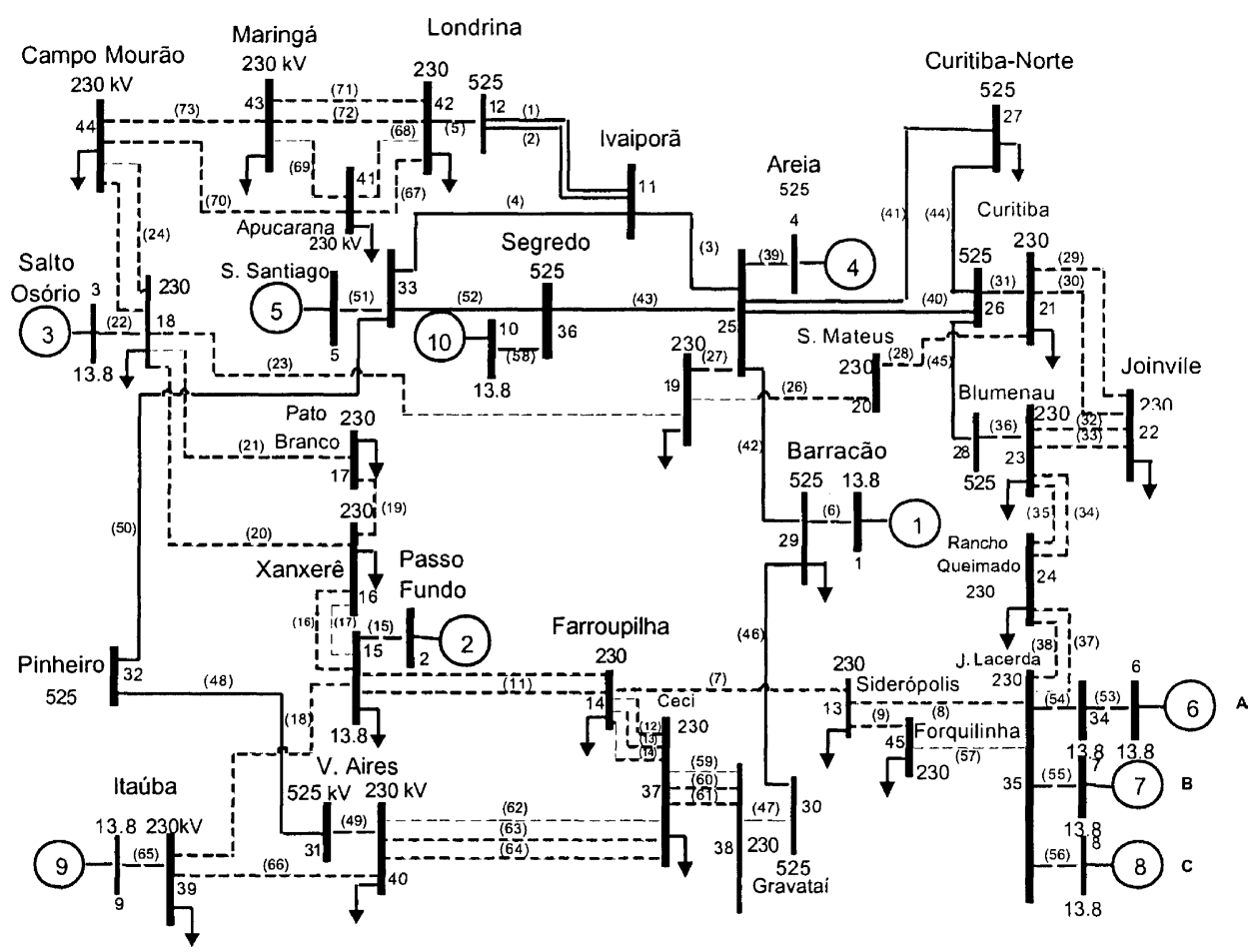

(--) Number of the transmission lines.

Fig. 4. Unifilar diagram of the electrical power system. 
Table 1

Set of contingencies

\begin{tabular}{llll}
\hline \multirow{2}{*}{$\begin{array}{l}\text { Number of } \\
\text { the fault }\end{array}$} & \multicolumn{2}{l}{ Fault } & \multicolumn{2}{l}{ Outage of circuits } \\
\cline { 2 - 4 } & Bus under short circuit & Initial bus & Final bus \\
\cline { 3 - 4 } & & 14 & 15 \\
\hline 1 & 15 & 16 & 18 \\
2 & 18 & 18 & 44 \\
3 & 18 & 29 & 30 \\
4 & 29 & 39 & 40 \\
5 & 39 & 16 & 16 \\
6 & 15 & 16 & 18 \\
7 & 16 & 16 & 17 \\
8 & 17 & 17 & 18 \\
9 & 17 & 33 & 36 \\
10 & 33 & 18 & 44 \\
11 & 44 & 15 & 16 \\
12 & 16 & 41 & 44 \\
13 & 44 & 13 & 35 \\
14 & 35 & 43 & 44 \\
15 & 44 &
\end{tabular}

Different generation and load profiles are considered (obtained with different percentile and different seeds), if compared to the data used on the training phase (Fig. 4).

The contingency set is shown in Table 1. Fifteen contingencies (arbitrarily chosen) are considered to illustrate the proposed methodology. However, this number can be increased according to the necessities of the user, with no problem in the formulation. In Table 2, the intervals of security margin (ISMs) are presented, adopting a representation of 4 bits, i.e. with $\vartheta_{\text {esp }}=0.35$ completing 15 intervals.

The neural network training is realized considering a set containing about 3000 generation and load profiles (in median 200 per contingency), and the corresponding security margins (for 15 contingencies).

In Table 3, it is shown the parameters used for the training phase of the fuzzy ART-ARTMAP neural network (Table 3).

After effectuating the training phase, the transient stability analysis can be realized, and the results are shown in Table 4.

Table 2

Intervals of the security margin

\begin{tabular}{ll}
\hline Number of the interval & Security margin interval (IMS) \\
\hline 1 & $(-\infty ;-3]$ \\
2 & $(-3 ;-1.9630]$ \\
3 & $(-1.9630 ;-1.1948]$ \\
4 & $(-1.1948 ;-0.6258]$ \\
5 & $(-0.6258 ;-0.2043]$ \\
6 & $(-0.2043 ; 0.10790]$ \\
7 & $(0.10790 ; 0.33920]$ \\
8 & $(0.33920 ; 0.51050]$ \\
9 & $(0.51050 ; 0.63740]$ \\
10 & $(0.63740 ; 0.73140]$ \\
11 & $(0.73140 ; 0.80110]$ \\
12 & $(0.80110 ; 0.85260]$ \\
13 & $(0.85260 ; 0.89080]$ \\
14 & $(0.89080 ; 0.91910]$ \\
15 & $(0.9191 ; 1]$ \\
\hline
\end{tabular}

Table 3

Specification of the neural network parameters

\begin{tabular}{ll}
\hline Item & Value \\
\hline Vigilance parameter & \\
$\rho a$ & 0.99 \\
$\rho b$ & 0.97 \\
$\rho a b$ & 0.90 \\
Training rate, $\beta$ & 1 \\
Chosen parameter, $\alpha$ & 0.1 \\
\hline
\end{tabular}

It is the comparative analysis with neural networks (proposed method) and the hybrid method [19] (considered in this work as correct values). The percentiles and the seeds used are shown in columns 2 and 3 of Table 4, respectively. The intervals ISMs, indicated by the fuzzy ART-ARTMAP neural network, correspond to the employment of the voting strategy [8], i.e. the neural network is trained many times (for example, five times), with different ordinations (random ordination) for the same set data of the training. The final prediction is given by the major frequency of the generated solutions. It is observed that the results produced by the two methodologies are very close.

Considering the results that were shown previously, it can be observed the following points:

(1) The total load of the system correspondent to the base case (100\% of the loading of the system) is $6655 \mathrm{MW}$.

(2) Thus, the generation and the load correspond to a variation interval between 5324 and $8651.5 \mathrm{MW}$.

(3) It is emphasized that the analysis phase is effectuated very fast. Therefore, there is compatibility for applications in real time. The training phase (activity realized off-line) spends much time of the processing time. However, this time is inferior, if compared to the other neural network available on the specialized literature.

(4) The neural network training was effectuated, for 15 contingencies, considering about 3000 pairs of pattern vectors (input/output), taking around $60 \mathrm{~s}$ ( $4 \mathrm{~s}$ per contingency). It is not included the time used for reading and writing the information. It is emphasized that the pre-processing of the data, for the neural network training, is effectuated by a module added to the program Simul. Therefore, the execution time of the Simul program and the pre-processing is not considered.

(5) The processing time for each analysis is about cents of seconds.

(6) Table 4 contains 138 transient stability analysis, considering the same contingencies used on the training phase, however, with different generation and load distribution, executed in a pseudo random way, seeking to simulate possible real operation cases. If the training is adequately realized, the analysis of real cases, is at first reliable.

(7) The obtained results can be considered satisfactory, however, some imprecision were observed (about 10\%). Such imprecision are not enough to invalidate the analysis. The error, is sometimes pessimist, i.e. it is found an ISM inferior to the real interval. This is probably due to the chosen 
Table 4

Results of the transient stability analysis obtained by simulation (reference results) and by fuzzy ART-ARTMAP neural network

\begin{tabular}{|c|c|c|c|c|}
\hline \multirow[t]{2}{*}{$\begin{array}{l}\text { Number of } \\
\text { the fault }\end{array}$} & \multicolumn{2}{|c|}{ Generation/load profile } & \multicolumn{2}{|c|}{$\begin{array}{l}\text { Security margin } \\
\text { interval (IMS) }\end{array}$} \\
\hline & $\begin{array}{l}\text { Percent of } \\
\text { base case }\end{array}$ & Seed & Correct & $\begin{array}{l}\text { Neural } \\
\text { network }\end{array}$ \\
\hline 1 & 75 & 123 & 15 & 15 \\
\hline 2 & 75 & 289 & 7 & 7 \\
\hline 3 & 75 & 73 & 9 & 9 \\
\hline 4 & 75 & 190 & 15 & 15 \\
\hline 5 & 75 & 391 & 13 & 13 \\
\hline 1 & 80 & 381 & 15 & 14 \\
\hline 2 & 80 & 73 & 9 & 9 \\
\hline 3 & 80 & 1 & 12 & 12 \\
\hline 4 & 80 & 355 & 15 & 15 \\
\hline 5 & 80 & 999 & 12 & 12 \\
\hline 1 & 85 & 24 & 12 & 12 \\
\hline 2 & 85 & 257 & 8 & 7 \\
\hline 3 & 85 & 191 & 9 & 8 \\
\hline 4 & 85 & 309 & 15 & 15 \\
\hline 5 & 85 & 60 & 8 & 8 \\
\hline 1 & 90 & 71 & 12 & 12 \\
\hline 2 & 90 & 137 & 5 & 5 \\
\hline 3 & 90 & 19 & 5 & 5 \\
\hline 4 & 90 & 244 & 15 & 15 \\
\hline 5 & 90 & 60 & 8 & 8 \\
\hline 6 & 90 & 458 & 15 & 15 \\
\hline 7 & 90 & 711 & 12 & 12 \\
\hline 8 & 90 & 711 & 13 & 12 \\
\hline 9 & 90 & 597 & 11 & 12 \\
\hline 10 & 90 & 597 & 12 & 11 \\
\hline 11 & 90 & 458 & 13 & 13 \\
\hline 12 & 90 & 273 & 12 & 12 \\
\hline 13 & 90 & 597 & 13 & 14 \\
\hline 14 & 90 & 597 & 15 & 15 \\
\hline 15 & 90 & 458 & 15 & 15 \\
\hline 1 & 95 & 200 & 12 & 12 \\
\hline 2 & 95 & 28 & 6 & 5 \\
\hline 3 & 95 & 163 & 5 & 5 \\
\hline 4 & 95 & 131 & 14 & 12 \\
\hline 5 & 95 & 2 & 6 & 6 \\
\hline 6 & 95 & 749 & 12 & 13 \\
\hline 7 & 95 & 391 & 12 & 12 \\
\hline 8 & 95 & 321 & 12 & 12 \\
\hline 9 & 95 & 497 & 13 & 13 \\
\hline 10 & 95 & 749 & 11 & 12 \\
\hline 11 & 95 & 123 & 14 & 14 \\
\hline 12 & 95 & 123 & 14 & 14 \\
\hline 13 & 95 & 321 & 14 & 14 \\
\hline 14 & 95 & 321 & 15 & 15 \\
\hline 15 & 95 & 458 & 15 & 15 \\
\hline 1 & 100 & - & 12 & 12 \\
\hline 2 & 100 & - & 5 & 5 \\
\hline 3 & 100 & - & 5 & 5 \\
\hline 4 & 100 & - & 15 & 15 \\
\hline 5 & 100 & - & 7 & 6 \\
\hline 6 & 100 & - & 12 & 12 \\
\hline 7 & 100 & - & 12 & 12 \\
\hline 8 & 100 & - & 12 & 12 \\
\hline 9 & 100 & - & 12 & 13 \\
\hline 10 & 100 & - & 12 & 12 \\
\hline 11 & 100 & - & 13 & 14 \\
\hline 12 & 100 & - & 13 & 14 \\
\hline 13 & 100 & - & 14 & 14 \\
\hline 14 & 100 & - & 12 & 12 \\
\hline 15 & 100 & - & 15 & 14 \\
\hline
\end{tabular}

Table 4 (Continued)

\begin{tabular}{|c|c|c|c|c|}
\hline \multirow[t]{2}{*}{$\begin{array}{l}\text { Number of } \\
\text { the fault }\end{array}$} & \multicolumn{2}{|c|}{ Generation/load profile } & \multicolumn{2}{|c|}{$\begin{array}{l}\text { Security margin } \\
\text { interval (IMS) }\end{array}$} \\
\hline & $\begin{array}{l}\text { Percent of } \\
\text { base case }\end{array}$ & Seed & Correct & $\begin{array}{l}\text { Neural } \\
\text { network }\end{array}$ \\
\hline 1 & 105 & 100 & 11 & 11 \\
\hline 2 & 105 & 389 & 4 & 4 \\
\hline 3 & 105 & 275 & 5 & 5 \\
\hline 4 & 105 & 380 & 15 & 15 \\
\hline 5 & 105 & 77 & 7 & 7 \\
\hline 6 & 105 & 109 & 12 & 12 \\
\hline 7 & 105 & 613 & 11 & 11 \\
\hline 8 & 105 & 931 & 11 & 12 \\
\hline 9 & 105 & 371 & 12 & 13 \\
\hline 10 & 105 & 61 & 11 & 12 \\
\hline 11 & 105 & 613 & 12 & 11 \\
\hline 12 & 105 & 371 & 13 & 13 \\
\hline 13 & 105 & 371 & 14 & 14 \\
\hline 14 & 105 & 613 & 8 & 8 \\
\hline 15 & 105 & 371 & 15 & 14 \\
\hline 1 & 110 & 121 & 11 & 11 \\
\hline 2 & 110 & 320 & 4 & 4 \\
\hline 3 & 110 & 11 & 5 & 5 \\
\hline 4 & 110 & 220 & 14 & 14 \\
\hline 5 & 110 & 55 & 7 & 7 \\
\hline 6 & 110 & 206 & 9 & 9 \\
\hline 7 & 110 & 365 & 11 & 12 \\
\hline 8 & 110 & 180 & 12 & 12 \\
\hline 9 & 110 & 439 & 9 & 10 \\
\hline 10 & 110 & 51 & 11 & 12 \\
\hline 11 & 110 & 365 & 11 & 11 \\
\hline 12 & 110 & 365 & 12 & 13 \\
\hline 13 & 110 & 180 & 14 & 14 \\
\hline 14 & 110 & 365 & 6 & 6 \\
\hline 15 & 110 & 439 & 15 & 14 \\
\hline 6 & 130 & 403 & 5 & 5 \\
\hline 6 & 125 & 30 & 6 & 5 \\
\hline 6 & 115 & 412 & 6 & 5 \\
\hline 6 & 110 & 206 & 9 & 9 \\
\hline 6 & 90 & 458 & 15 & 15 \\
\hline 7 & 130 & 403 & 9 & 8 \\
\hline 7 & 125 & 30 & 9 & 8 \\
\hline 7 & 120 & 611 & 8 & 8 \\
\hline 7 & 115 & 350 & 8 & 8 \\
\hline 7 & 110 & 519 & 11 & 11 \\
\hline 8 & 130 & 121 & 8 & 9 \\
\hline 8 & 127.5 & 288 & 13 & 13 \\
\hline 8 & 122.5 & 240 & 12 & 11 \\
\hline 8 & 117.5 & 163 & 13 & 13 \\
\hline 8 & 112.5 & 360 & 12 & 12 \\
\hline 9 & 130 & 3 & 6 & 7 \\
\hline 9 & 127.5 & 288 & 12 & 12 \\
\hline 9 & 122.5 & 240 & 11 & 11 \\
\hline 9 & 117.5 & 805 & 12 & 12 \\
\hline 9 & 112.5 & 71 & 10 & 10 \\
\hline 10 & 130 & 832 & 10 & 11 \\
\hline 10 & 127.5 & 77 & 9 & 9 \\
\hline 10 & 122.5 & 425 & 10 & 11 \\
\hline 10 & 117.5 & 819 & 9 & 9 \\
\hline 10 & 112.5 & 71 & 9 & 9 \\
\hline 11 & 130 & 220 & 11 & 12 \\
\hline 11 & 127.5 & 190 & 11 & 8 \\
\hline 11 & 122.5 & 867 & 9 & 8 \\
\hline 11 & 117.5 & 515 & 10 & 10 \\
\hline 11 & 112.5 & 500 & 11 & 11 \\
\hline 12 & 130 & 121 & 9 & 9 \\
\hline & & & & \\
\hline
\end{tabular}


Table 4 (Continued)

\begin{tabular}{|c|c|c|c|c|}
\hline \multirow[t]{2}{*}{$\begin{array}{l}\text { Number of } \\
\text { the fault }\end{array}$} & \multicolumn{2}{|c|}{ Generation/load profile } & \multicolumn{2}{|c|}{$\begin{array}{l}\text { Security margin } \\
\text { interval (IMS) }\end{array}$} \\
\hline & $\begin{array}{l}\text { Percent of } \\
\text { base case }\end{array}$ & Seed & Correct & $\begin{array}{l}\text { Neural } \\
\text { network }\end{array}$ \\
\hline 12 & 122.5 & 333 & 11 & 11 \\
\hline 12 & 117.5 & 515 & 10 & 9 \\
\hline 12 & 112.5 & 360 & 12 & 13 \\
\hline 13 & 130 & 121 & 9 & 8 \\
\hline 13 & 127.5 & 77 & 13 & 13 \\
\hline 13 & 122.5 & 380 & 12 & 13 \\
\hline 13 & 117.5 & 131 & 12 & 13 \\
\hline 13 & 112.5 & 500 & 13 & 13 \\
\hline 14 & 130 & 3 & 1 & 1 \\
\hline 14 & 127.5 & 349 & 1 & 1 \\
\hline 14 & 122.5 & 240 & 1 & 1 \\
\hline 14 & 117.5 & 163 & 1 & 1 \\
\hline 14 & 112.5 & 383 & 6 & 6 \\
\hline 15 & 130 & 365 & 15 & 14 \\
\hline 15 & 127.5 & 77 & 15 & 13 \\
\hline 15 & 122.5 & 603 & 15 & 13 \\
\hline 15 & 117.5 & 251 & 15 & 14 \\
\hline 15 & 112.5 & 385 & 15 & 14 \\
\hline
\end{tabular}

criterion of the fuzzy algorithm ( $T$ function of Step 4 from Section 4.6): when there is a draw in choosing the winner neuron, the option is effectuated taking the neuron with the minimum index.

\section{Conclusion}

It is developed in this work, a proceeding for multi-machine electrical energy transient stability analysis, by fuzzy ARTARTMAP neural network. With this architecture, it can be idealized a proceeding for electric power system transient stability analysis. The neural network input stimulus is constituted of the active and reactive nodal vectors (analog data) and the contingency data (binary data). The outputs correspond to the security margins of the system, represented by intervals comprehended between the minimum and the maximum values. This way, it is possible to represent the outputs in binary code (binary output). As an illustration of the methodology of the transient stability analysis by fuzzy ART-ARTMAP neural network [7], results are presented considering an electrical power system corresponding to a system (a south Brazil system version) composed of 45 busses, 72 transmission lines/transformers and 10 synchronous machines. The training is realized considering 15 contingencies of a solid three phase short circuit with outage of transmission line. The contingencies are arbitrated as incidents in several points of the electrical network ( 15 localization). The load levels are considered arbitrated in a random way in a universe comprehended between 80 and $130 \%$ of the total load of the system. The obtained results are satisfactory, i.e. they attained the objectives proposed. The objectives are to investigate the application of neural network for executing diagnosis of electrical power system transient stability. The ART-ARTMAP neural network provides very fast solutions (about cents of seconds, using a Pentium III of $500 \mathrm{MHz}$ microcomputer, considering the execu- tion of the voting strategy with five repetitions of the training). The quality of the solutions is adequate. However, imprecisions are observed. These imprecisions are generated in part by the conception of the ART family neural network, that needs to be continuously improved, and the use of a training set not sufficiently complete. The precision of the results is dependent of the volume of the data used on the training, and also on the number of the output segments (ISM intervals). A great number of segments can be obtained taking values less than the $\vartheta$ parameter. However, to increase the number of intervals it is necessary to use a great set of training pairs (number of examples) to reduce the possibilities of the existence of empty intervals. Empty intervals (occurrence of no security margin values in determined intervals, considering the training data) do not offer opportunity of learning and, consequently, introduce focus of noise on the training. More segmentation requires more number of pattern vectors on the training. Concluding, the fuzzy ART-ARTMAP neural network obtains very fast answers (there is compatibility with applications on-line) and the precision is dependent of the implementation of a schema adequate to the training adopting a volume of input and output well elaborated data (either in quality or enough quantity). It is emphasized that, the question referred to the quantity of data, can be solved by a faster training realized as proposed in this work, and the incorporation of continuous training module. The neural network, with this conception, can give better results with the passing years. The ART-ARTMAP neural network used in this work, is characterized as a viable tool to analyze electrical power system transient stability. This approach can be more optimized (aiming to proportionate velocity and precision for the solutions) including improvements by proposing new architectures. For example, references [7], (where it was proposed a new architecture of the ART neural network called fuzzy ART-ARTMAP neural network) and [9], where were introduced important improvements, profiting of the characteristic of malleability of the ascendant ART neural networks. New propositions can still be implemented, depending on the ability of the researches.

\section{References}

[1] B. Stott, Power system dynamic response calculations, in: Proceedings of the IEEE, vol. 67, no. 2, 1979, pp. 219-241.

[2] M.A. Pai, Power System stability: analysis by the direct method of Lyapunov, in: North-Holland Control Series, North-Holland Publishing Company, New York, 1981.

[3] P.M. Anderson, A.A. Fouad, Power System Control and Stability, IEEE Series on Power Engineering, second ed., USA, 2003.

[4] M. Pavella, D. Ernst, D. Ruiz-Vega, Transient Stability of Power Systems: A Unified Approach to Assessment and Control, Klüwer Academic Publishers, London, 2000.

[5] S.V. Kartalopoulos, Understanding Neural Networks and Fuzzy Logic, IEEE Press, New York, 1996.

[6] P.J. Werbos, Beyond Regression: New Tools For Prediction and Analysis in the Behavioral Sciences, Master Thesis, Harvard University, 1974.

[7] W.P. Ferreira, Análise de Segurança de Sistemas de Energia Elétrica Por Redes Neurais Baseadas na Teoria de Ressonância Adaptativa, Exame de Qualificação de Doutorado, Programa de Pós-graduação em Engenharia Elétrica-UNESP, Ilha Solteira-SP, 2003.

[8] G.A. Carpenter, S. Grossberg, N. Markuzon, J.H. Reynolds, D.B. Rosen, Fuzzy ARTMAP: a neural network architecture for incremental super- 
vised learning of analog multidimensional maps, IEEE Trans. Neural Netw. 3 (5) (1992) 698-713.

[9] E. Granger, M.A. Rubin, S. Grossberg, P.A. Lavoie, What-and-where fusion neural network for recognition and tracking of multiple radar emitters, Neural Netw. (14) (2001) 325-344.

[10] D.J. Sobajic, Y.H. Pao, Artificial neural-net based dynamic security assessment for electric power systems, IEEE Trans. Power Syst. 4 (1) (1989) 220-227.

[11] Y.H. Pao, D.J. Sobajic, Combined use of unsupervised and supervised learning for dynamic security assessment, IEEE PICA-91 (1991) 278-284.

[12] C.R. Minussi, M.C.G. Silveira, Transient stability analysis of electric power systems by neural networks, in: 38rd Midwest Symposium on Circuits and Systems, Rio de Janeiro-RJ, 1995, pp. 1305-1308.

[13] C.R. Minussi, L.M. Ramos, S.C. Marchiori, M.L.M. Lopes, A.D.P Lotufo, Neural networks to transient stability analysis of electrical power systems, in: Proceedings of the V Brazilian Conference on Neural Networks, PUC, Rio de Janeiro-RJ, 2001, pp. 31-36.

[14] M.C.G. Silveira, A.D.P. Lotufo, C.R. Minussi, Transient stability analysis of electrical power systems using a neural network based on fuzzy ARTMAP, in: IEEE Bologna Power Tech 2003 Conference, Bologna, Italy, 2003.
[15] S.C. Marchiori, C.R. Minussi, A.D.P. Lotufo, Rede Neural ARTMAP Nebulosa Para Análise de Estabilidade Transitória de Sistemas de Energia Elétrica, Learn. Nonlin. Models 1 (1) (2002) 61-78.

[16] M.C.G. Silveira, S.C. Marchiori, W.P. Ferreira, C.R. Minussi, Análise de Estabilidade Transitória de Sistemas de Energia Elétrica Usando Uma Rede Neural ARTMAP Nebulosa, in: XIV Congresso Brasileiro de Automática (CBA 2002), Natal-RN, 2002, pp. 31713177.

[17] C.R. Minussi, W. Freitas Filho, Sensitivity analysis for transient stability, in: IEEE Proceedings on Generation, Transmission and Distribution, vol. 145, no. 6, 1998, pp. 669-674.

[18] T. Athay, V.R. Sherket, R. Podmore, S. Virmani, C. Puech, Transient energy stability analysis, in: Conference on 'System Engineering for Power', Davos, Switzerland, 1979.

[19] L.G.S. Fonseca, I.C. Decker, Iterative algorithm for critical energy determination in transient stability of power system, in: IFAC-Symposium Planning and Operation in Electric Energy System, RJ-Brazil, 1985, pp. 483-489.

[20] M.C.G. Silveira, Formulação do Problema da Análise de Estabilidade Transitória Por Redes Neurais ARTMAP Nebulosas, Exame Geral de Qualificação de Doutorado, Programa de Pós-graduação em Engenharia Elétrica-UNESP, Ilha Solteira-SP, 2003 (in Portuguese). 